\title{
High expression of ETS2 predicts poor prognosis in acute myeloid leukemia and may guide treatment decisions
}

\author{
Lin $\mathrm{Fu}^{1,2,3 \dagger}$, Huaping $\mathrm{Fu}^{4 \dagger}$, Qingyun $\mathrm{Wu}^{2 \dagger}$, Yifan Pang ${ }^{5}$, Keman Xu' ${ }^{6}$, Lei Zhou ${ }^{7}$, Jianlin Qiao ${ }^{2}$, Xiaoyan Ke ${ }^{*}$, \\ Kailin $\mathrm{Xu}^{2^{*}}$ and Jinlong Shi ${ }^{3,8,9^{*}}$
}

\begin{abstract}
Background: ETS2 is a downstream effector of the RAS/RAF/ERK pathway, which plays a critical role in the development of malignant tumor. However, the clinical impact of ETS2 expression in AML remains unknown.

Methods: In this study, we evaluated the prognostic significance of ETS2 expression using two relatively large cohorts of AML patients.

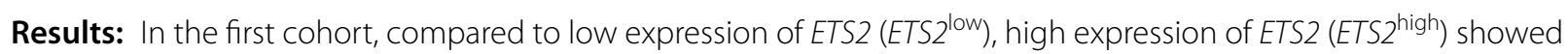
significant shorter OS, EFS and RFS in the current treatments including the allogeneic HCT group $(n=72)$ and the chemotherapy group $(n=100)$. Notably, among $E_{S} 2^{\text {high }}$ patients, those received allogeneic HCT had longer OS, EFS and RFS than those with chemotherapy alone (allogeneic HCT, $n=39$ vs. chemotherapy, $n=47$ ), but treatment modules play insignificant role in the survival of ETS2 ${ }^{\text {low }}$ patients (allogeneic HCT, $n=33$ vs. chemotherapy, $n=53$ ). Moreover, gene/microRNA expression data provides insights into the biological changes associated with varying ETS2 expression levels in AML. The prognostic value of ETS2 was further validated in the second AML cohort $(n=329)$.
\end{abstract}

Conclusions: Our results indicate that ETS2 ${ }^{\text {high }}$ is a poor prognostic factor in AML and may guide treatment decisions towards allogeneic HCT.

Keywords: ETS2, Prognosis, AML, Allogeneic HCT

\section{Background}

Acute myeloid leukemia (AML) represents a group of myeloid malignancies with remarkably heterogeneous outcomes [1]. Finding effective prognostic biomarkers has been one of the most urgent clinical needs and research hotspots. So far, a few prognosticators have been established, including mutations in NPM1 and double $C E B P A$ that are associated with favourable outcomes;

\footnotetext{
*Correspondence: jinlong_301@163.com; xiaoyank@yahoo.com; lihmd@163.com

${ }^{\dagger}$ Lin Fu, Huaping Fu and Qingyun Wu contributed equally to this work ${ }^{1}$ Department of Hematology and Lymphoma Research Center, Peking University, Third Hospital, Beijing 100191, China

${ }^{2}$ Department of Hematology, The Affiliated Hospital of Xuzhou Medical University, Xuzhou 221002, China

${ }^{3}$ Department of Hematology, Huaihe Hospital of Henan University, Kaifeng 475000, China

Full list of author information is available at the end of the article
}

whereas FLT3-ITD is associated with poor prognosis. High expression levels of WT1 [2], miR-155 [3, 4], ERG [5, 6], BAALC [6], and MN1 [7] have also been shown to be poor prognostic factors in AML.

V-ets avian erythroblastosis virus E26 oncogene homolog 2 (ETS2), a downstream target for both the Ras/ Raf/MAP kinase and phosphatidylinositol 3-kinase/Akt pathways. ETS2 is one of the founder members of the E26 transformation-specific (ETS) family located on human chromosome 21 [8]. ERG, one of the classic prognostic markers in AML, also belongs to the ETS family. ETS2 and ERG had been shown to be overexpressed in AML patients with complex karyotypes involving chromosome 21 [9]. Although ETS2 was initially characterized as a proto-oncogene acute megakaryocytic leukemia (AMKL) [10], however, the clinical impact of ETS2 expression in AML remains unknown. 
In recent years, many studies suggest that ETS 2 exhibit both tumor-promoting and tumor-suppressive effects in malignancies. For example, ETS2 has been found to be an oncogene in patients with AML [11], but it also has tumorsuppressive effects in non-small cell lung cancer [12]. Here, we demonstrate ETS2 $2^{\text {high }}$ as an adverse prognostic biomarker for AML based on analysis of two separate datasets and indicate ETS $2^{\text {high }}$ may guide treatment decisions towards allogeneic HCT; we also explore the distinctive gene/microRNA patterns associated with ETS2 expression.

\section{Methods}

\section{Patients}

The first cohort was derived from The Cancer Genome Atlas (TCGA) dataset, including 200 clinically annotated adult de novo AML samples [13]. In this cohort, RNA sequencing for 179 samples and microRNA sequencing for 194 samples had been previously reported. Detailed descriptions of clinical and molecular characteristics were also provided. All these data were publicly accessible from the TCGA website. The study was approved by the human studies committee at Washington University with written informed consent obtained from all patients.

The second cohort was derived from a whole AML cohort ( $\mathrm{n}=329$ ) diagnosed and collected at Erasmus University Medical Center (Rotterdam) between 1990 and 2008, approved by the institutional review boards at Weill Cornell Medical College and Erasmus University Center, and all subjects provided written informed consent in accordance with the Declaration of Helsinki. Microarray expression profiles were obtained by Affymetrix Human Genome 133 plus 2.0 and U133A Gene Chips from GSE6891 data. All experiments' design, quality control and data normalization were in line with the standard Affymetrix protocols. All clinical, cytogenetic and molecular information as well as microarray data of these patients were publicly accessible at the Gene Expression Omnibus (GSE6891, http://www.ncbi.nlm.nih.gov/geo) [14]. All patients were uniformly treated under the study protocols of Dutch-Belgian Cooperative Trial Group for Hematology Oncology (HOVON, details of therapeutic protocol available at http://www.hovon.nl).

\section{Statistical analyses}

OS was defined as the time from the date of diagnosis to death due to any cause. EFS was defined as the time from the date of diagnosis to removal from the study due to the absence of complete remission, relapse or death. RFS was defined as the time from the date of diagnosis to removal from the study due to relapse.

Patients with higher than median ETS2 expression values of all patients were classified as ETS $2^{\text {high }}$, and those with lower than median expression values were classified as ETS2 $2^{\text {low }}$. To investigate the associations between ETS2 expression levels and clinical, molecular characteristics, the Fisher exact and Wilcoxon rank-sum tests were used for hypothesis testing with categorical and continuous variables, respectively. The associations between ETS2 expression and the OS, EFS and RFS were analyzed by the Kaplan-Meier method and the log-rank test. Multivariate Cox proportional hazard models were employed to study the associations between ETS2 expression levels and OS, EFS and RFS in the presence of other known risk factors. Student's $t$ test and multiple hypothesis correction (False Discovery Rate, FDR) was used to identify different gene/ microRNA between ETS $2^{\text {high }}$ and ETS2 ${ }^{\text {low }}$ groups. The statistical cutoff values were an absolute fold-change (FC) $\geq 1.5$ and an adjusted $P$ value $\leq 0.05$. All analyses were performed by the R 3.1.1 software packages.

\section{Results}

Expression of ETS2 in AML patients and normal controls

A microarray dataset of bone marrow (BM) samples was used for differential expression analysis, including 30 AML BM and 17 normal BM (NBM) samples (GSE37307, http://www.ncbi.nlm.nih.gov/geo), and 62 AML BM and 42 NBM samples (GSE63270, http://www.ncbi.nlm.nih. gov/geo). Higher expression of ETS2 was shown significantly in AML BM than NBM $(P=0.01$, Fig. 1 a and $P=0.05$, Fig. 1b).

\section{Relative expression of ETS2 in different National Comprehensive Cancer Network (NCCN) risk subgroups} In the first cohort, ETS2 showed averagely higher expression in the NCCN poor- and intermediate-risk patients than that in the good-risk group (good vs. intermediate $P=0$, intermediate vs. poor $P=0.0181$, and good vs. poor $P=0$, respectively; Fig. $1 c)$.

\section{Associations between ETS2 expression and other classic prognostic biomarkers in AML}

The first cohort were further divided into subgroups by the presence of FLT3-ITD and mutation status of NPM1 and CEBPA. Levels of ETS2 expression were compared among different subgroups. ETS2 showed significantly higher expression in samples with FLT3-ITD compared than samples without FLT3-ITD $(P=0.006$, Fig. $1 \mathrm{~d})$. No significant differences were revealed between NPM1mutated and wild-type samples $(P=0.0657)$ or between CEBPA-mutated and wild-type samples $(P=0.2977$, Fig. 1d).

\section{Differences in clinical and molecular characteristics between $E T S 2^{\text {high }}$ and ETS2 ${ }^{\text {low }}$ groups}

In the first cohort, ETS $2^{\text {high }}$ patients were more likely to be $\geq 60$-year-old, and had higher WBC count, higher 

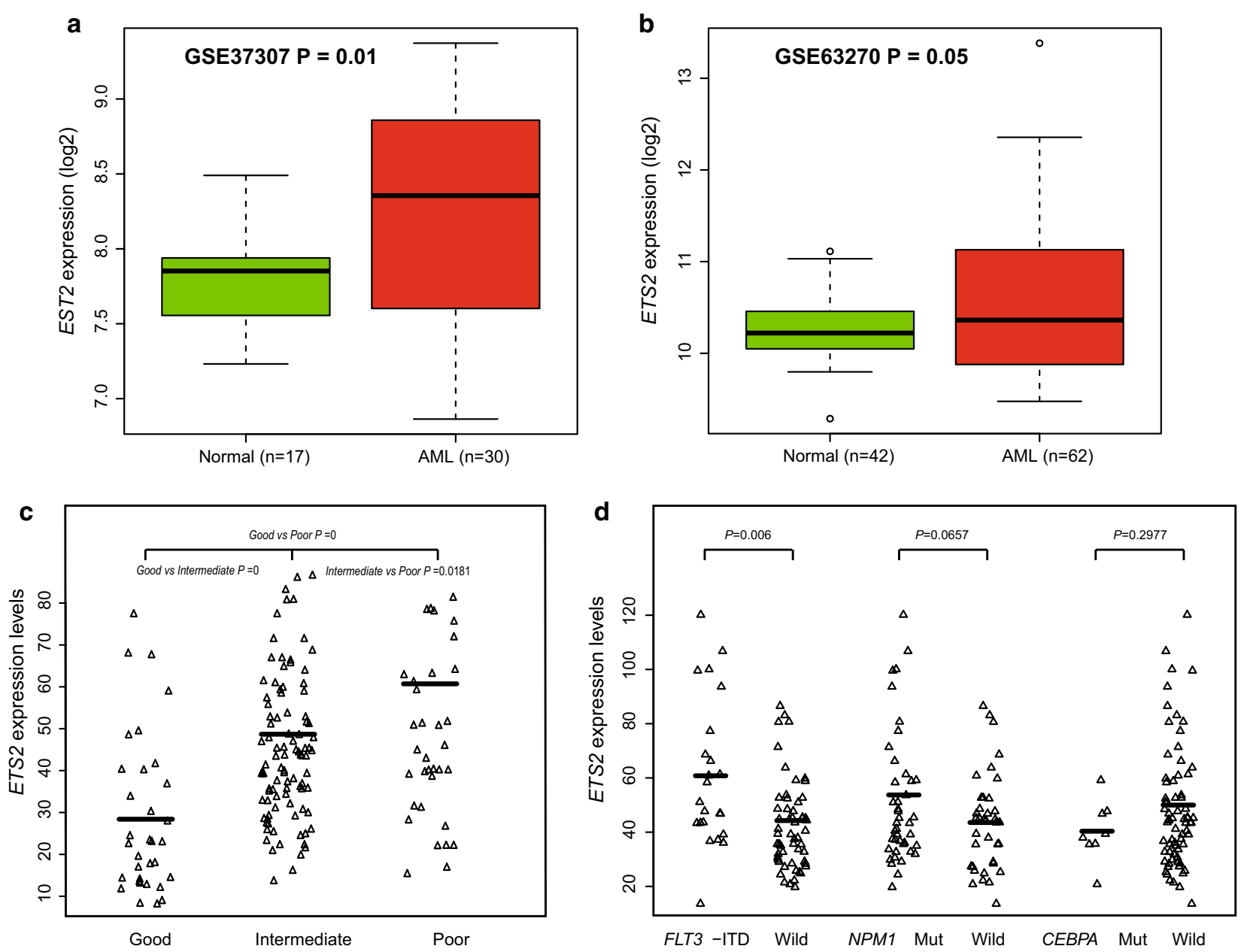

Fig. 1 Differences in the expression of ETS2 in AML. a AML-BM cases $(n=30)$ compared with NBM samples $(n=17)$, b AML-BM cases $(n=62)$ compared with NBM samples $(n=42)$, c relative expression of ETS2 in the different NCCN-risk subgroup (good, intermediate and poor) of AML cases, $\mathbf{d}$ associations between ETS2 expression and other classic prognostic biomarkers in AML cases (FLT3-ITD and the mutation of NPM1 and CEBPA)

peripheral blood blasts, more diagnosed with M0, M1, M3, or M5 FAB subtypes, and more FLT3-ITD and TP53 mutation $(P=0.004, P=0.05, P=0.01, P=0.001$, $P=0.005, P<0.001, P=0.04, P=0.01, P=0.016$, respectively) comparing with $E T S 2^{\text {low }}$ patients. No other associations between ETS2 expression and other mutations were found. Additionally, ETS2 ${ }^{\text {high }}$ patients with AML were more likely to have a higher expression of $M N 1$, miR155HG and WT1 than ETS2 ${ }^{\text {low }}$ patients $(P=0.04$, $P<0.001$, and $P=0.009$, respectively). See Table 1 .

\section{ETS2 ${ }^{\text {high }}$ was associated with adverse outcomes}

ETS2 ${ }^{\text {high }}$ patients had markedly shorter OS (Fig. 2a, $P=2 \mathrm{e}^{-6}$ ), EFS (Fig. $2 \mathrm{~b}, P=1 \mathrm{e}^{-6}$ ) and RFS (Fig. 2c, $\left.P=3.8 \mathrm{e}^{-5}\right)$ comparing with $E T S 2^{\text {low }}$ patients. Associations between ETS2 expression and prognostic significance within the allogeneic HCT group and chemotherapy group were also separately analyzed. Within the allogeneic HCT group $(\mathrm{n}=72)$, significant differences were observed in OS (Fig. $2 \mathrm{~d}, P<0.001$ ), EFS (Fig. $2 \mathrm{e}, P=0.002$ ) and RFS (Fig. 2f, $P=0.012$ ) between the ETS2 $2^{\text {high }}$ and ETS2 $2^{\text {low }}$ patients. In the chemotherapy group $(\mathrm{n}=99), E T S 2^{\text {high }}$ patients had significantly shorter OS (Fig. $2 \mathrm{~d}, P<0.001$ ), EFS (Fig. 2e, $P<0.001$ ) and RFS (Fig. 2f, $P=0.002$ ) than $E T S 2^{\text {low }}$ patients. Moreover, ETS2 $2^{\text {high }}$ patients who received allogeneic $\mathrm{HCT}$ had significantly longer OS and EFS than chemotherapy-only (OS, $P<0.002$; EFS, $P=0.029$, respectively), whereas treatment modules play insignificant role in the survival of $E T S 2^{\text {low }}$ patients (allogeneic HCT vs. chemotherapy-only; OS, $P=0.067$; EFS, $P=0.774 ;$ RFS, $P=0.148$, respectively).

\section{ETS2 expression was associated with shorter OS, EFS and RFS in multivariate analyses}

To adjust for the impact of known clinical and molecular risk factors, we performed multivariate analyses to 
Table 1 Comparison of clinical and molecular characteristics of de novo AML patients according to ETS2

\begin{tabular}{|c|c|c|c|}
\hline \multirow[t]{2}{*}{ Variable } & \multicolumn{3}{|c|}{ AML (TCGA dataset) } \\
\hline & ETS2 $^{\text {high }}(n=89)$ & $\mathrm{ETS}^{\text {low }}(\mathrm{n}=90)$ & $\mathbf{P}$ \\
\hline Median age, year (range) & & & 0.01 \\
\hline Median & 61 & 54.5 & \\
\hline Range & $(18-88)$ & $(22-82)$ & \\
\hline Age group, n (\%) & & & 0.004 \\
\hline$<60$ & 38 & 58 & \\
\hline$\geq 60$ & 51 & 32 & \\
\hline WBC count, $X 10^{9} / \mathrm{L}$ & & & 0.05 \\
\hline Median & 33.2 & 12.2 & \\
\hline Range & $0.6-297.4$ & $0.4-202.7$ & \\
\hline BM blasts (\%) & & & 0.6 \\
\hline Median & 74 & 72 & \\
\hline Range & $32-100$ & $30-100$ & \\
\hline PB blasts (\%) & & & 0.01 \\
\hline Median & 49 & 25 & \\
\hline Range & $0-98$ & $0-97$ & \\
\hline \multicolumn{4}{|l|}{ FAB subtype, no (\%) } \\
\hline Mo & 14 & 2 & 0.001 \\
\hline M1 & 30 & 14 & 0.005 \\
\hline $\mathrm{M} 2$ & 18 & 22 & 0.59 \\
\hline M3 & 0 & 16 & $<0.001$ \\
\hline M4 & 16 & 19 & 0.6 \\
\hline M5 & 6 & 15 & 0.04 \\
\hline M6 & 1 & 1 & 1 \\
\hline Others & 4 & 1 & 0.21 \\
\hline FLT3-ITD, n (\%) & & & 0.01 \\
\hline Present & 25 & 12 & \\
\hline Absent & 64 & 78 & \\
\hline $\begin{array}{l}\text { NPM1 (no FLT3-ITD), } \\
\text { n (\%) }\end{array}$ & & & 0.33 \\
\hline Mutated & 12 & 17 & \\
\hline Wild-type & 77 & 73 & \\
\hline CEBPA, n (\%) & & & 0.24 \\
\hline Single mutated & 3 & 5 & \\
\hline Double mutated & 1 & 4 & \\
\hline Wild-type & 85 & 81 & \\
\hline MLL-PTD, n (\%) & & & 0.33 \\
\hline Mutated & 6 & 3 & \\
\hline Wild-type & 83 & 87 & \\
\hline IDH1, n (\%) & & & 0.58 \\
\hline Mutated & 9 & 7 & \\
\hline Wild-type & 80 & 83 & \\
\hline IDH2, n (\%) & & & 0.78 \\
\hline Mutated & 9 & 8 & \\
\hline Wild-type & 80 & 82 & \\
\hline RUNX1, n (\%) & & & 0.08 \\
\hline Mutated & 12 & 5 & \\
\hline Wild-type & 77 & 85 & \\
\hline
\end{tabular}

Table 1 continued

\begin{tabular}{|c|c|c|c|}
\hline \multirow[t]{2}{*}{ Variable } & \multicolumn{3}{|c|}{ AML (TCGA dataset) } \\
\hline & ETS2 $^{\text {high }}(n=89)$ & $\operatorname{ETS}^{\text {low }}(n=90)$ & $\mathbf{P}$ \\
\hline DNMT3A, n (\%) & & & 0.21 \\
\hline R882 mutated & 16 & 7 & \\
\hline Non-R822 mutated & 9 & 11 & \\
\hline Wild-type & 64 & 72 & \\
\hline TP53, n (\%) & & & 0.016 \\
\hline Mutated & 12 & 3 & \\
\hline Wild-type & 77 & 87 & \\
\hline ERG expression, n (\%) & & & 0.16 \\
\hline High & 49 & 40 & \\
\hline Low & 40 & 50 & \\
\hline BAALC expression, n (\%) & & & 0.07 \\
\hline High & 50 & 39 & \\
\hline Low & 39 & 51 & \\
\hline MN1 expression, n (\%) & & & 0.04 \\
\hline High & 51 & 38 & \\
\hline Low & 38 & 52 & \\
\hline $\begin{array}{l}\text { miR155HG expression, } \\
\text { n (\%) }\end{array}$ & & & $<0.001$ \\
\hline High & 56 & 33 & \\
\hline Low & 33 & 57 & \\
\hline WT1 expression, n (\%) & & & 0.009 \\
\hline High & 53 & 36 & \\
\hline Low & 36 & 54 & \\
\hline
\end{tabular}

High ERG, BAALC, MN1, miR155HG and WT1 expression were defined as an expression level above the median of all samples, respectively

FAB French-American-British classification, FLT3-ITD internal tandem duplication of the FLT3 gene, MLL-PTD partial tandem duplication of the MLL gene

confirm the prognostic significance of ETS2 expression (Table 2). In the multivariate models for OS, EFS and RFS, ETS $2^{\text {high }}$ had adverse impacts on OS $(P=0.002)$, EFS $(P<0.001)$ as well as RFS $(P<0.001)$. Age was the only other factor negatively correlated with OS $(P<0.001)$ and EFS $(P<0.001)$.

\section{Associations between genome-wide gene-expression profiles and ETS2 expression}

To further assess the role of ETS2 in AML, we derived ETS2-associated gene expression profiles by high throughput sequencing from TCGA data. We first identified 368 up-regulated and 171 down-regulated genes that were significantly associated with ETS2 expression $(P<0.05$, fold change $=1.5$, Fig. $3 \mathrm{a})$. With a more rigorous analysis (fold change $=2$, and profiles without applicable values were all deleted), 359 genes were filtered out and the rest 180 genes were presented in an aberrant expression heat map (Fig. 3b).

Many genes known as unfavorable biomarkers were up-regulated, including leukemia-associated molecules, 


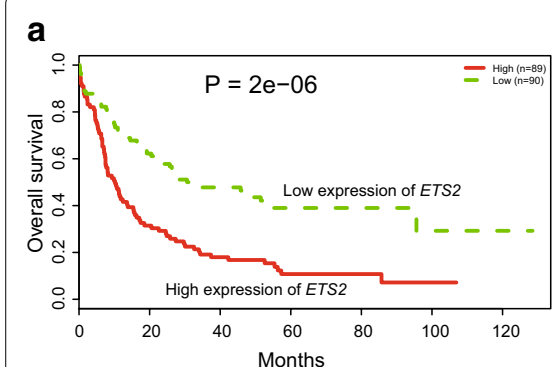

b
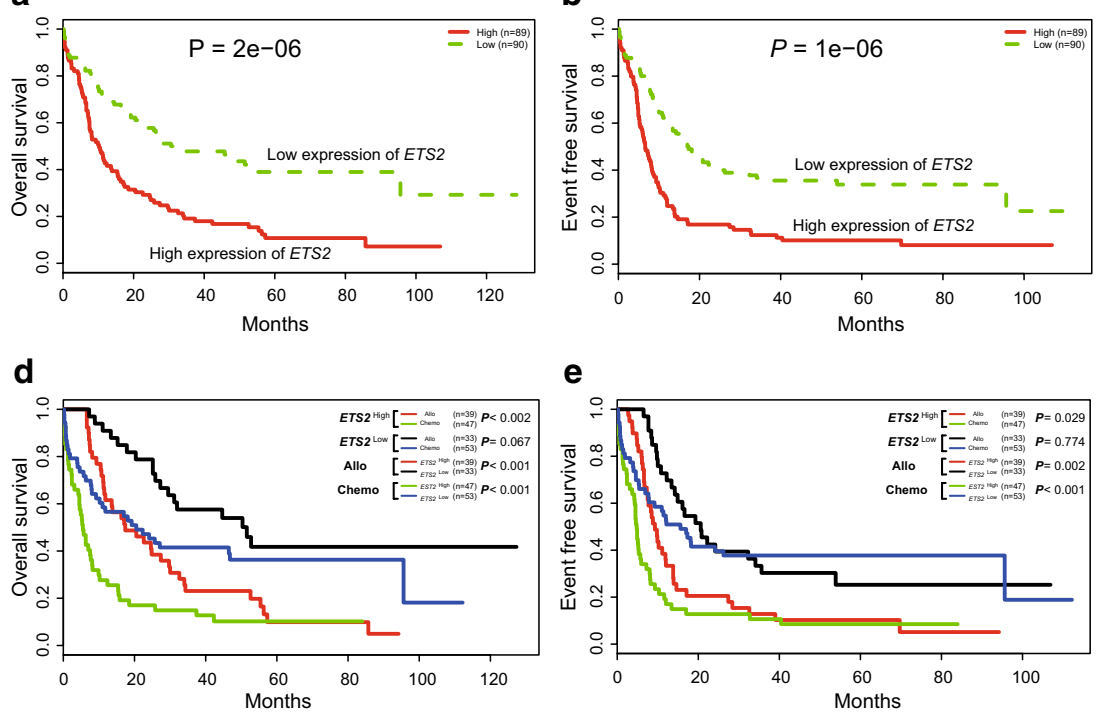

e

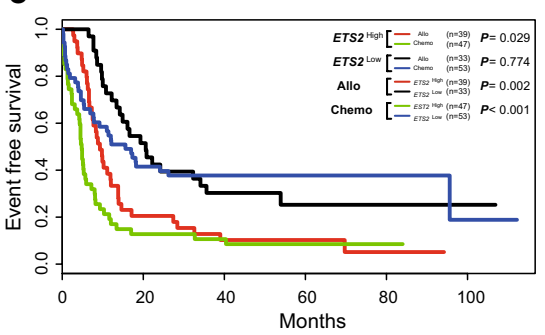

C

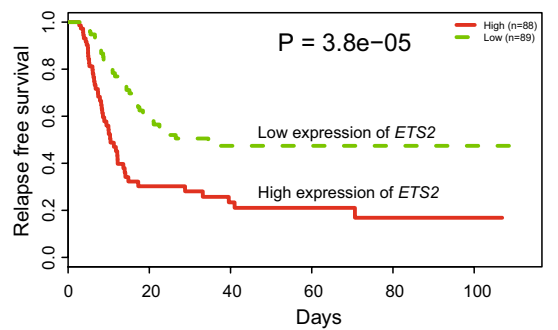

$\mathbf{f}$

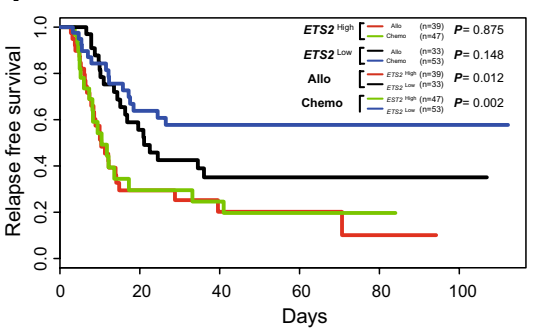

Fig. 2 The prognostic value of ETS2 expression in AML patients from TCGA data. a OS and $\mathbf{b}$ EFS and $\mathbf{c}$ RFS of the entire AML patients $(\mathrm{n}=179)$. d OS and $\mathbf{e}$ EFS and $\mathbf{f}$ RFS of the AML patients of ETS2 ${ }^{\text {high }}$ group $(n=86)$, ETS2 ${ }^{\text {low }}$ group $(n=88)$, allogeneic HCT group $(n=72)$ and chemotherapyonly group $(n=100)$. Allo allogeneic HCT, Chemo chemotherapy

Table 2 Multivariable analysis with OS and EFS in the primary cohort of 179 AML patients (TCGA dataset)

\begin{tabular}{llcc}
\hline Variables in final model by end point & HR & $\mathbf{9 5 \%} \mathbf{C l}$ & $\boldsymbol{P}$ value \\
\hline OS (all AML, $\mathrm{n}=179$ ) & & & \\
ETS2 expression, high vs. low & 1.79 & $1.23-2.59$ & 0.002 \\
Age, per 10-year increase & 1.46 & $1.27-1.68$ & $<0.001$ \\
CEBPA mutation vs. wild & 1.75 & $0.85-3.58$ & 0.13 \\
NPM1 mutation vs. wild & 1.1 & $0.73-1.66$ & 0.65 \\
FLT3-ITD, presented vs. others & 1.24 & $0.78-1.96$ & 0.37 \\
EFS (all AML, $\mathrm{n}=179$ ) & & & \\
ETS2 expression, high vs. low & 1.88 & $1.32-2.68$ & $<0.001$ \\
Age, per 10-year increase & 1.34 & $1.18-1.53$ & $<0.001$ \\
CEBPA mutation vs. wild & 1.2 & $0.92-3.57$ & 0.08 \\
NPM1 mutation vs. wild & 1.2 & $0.83-1.78$ & 0.3 \\
FLT3-ITD, presented vs. others & 1.4 & $0.9-2.15$ & 0.1 \\
RFS (all AML, $\mathrm{n}=177$ ) & & & \\
ETS2 expression, high vs. low & 2.23 & $1.41-3.5$ & $<0.001$ \\
Age, per 10-year increase & 1.13 & $0.96-1.33$ & 0.14 \\
CEBPA mutation vs. wild & 0.4 & $0.94-4.48$ & 0.07 \\
NPM1 mutation vs. wild & 0.25 & $0.81-2.15$ & 0.26 \\
FLT3-ITD, presented vs. Others & 1.47 & $0.86-2.53$ & 0.16 \\
\hline
\end{tabular}

OS overall survival, EFS event-free survival, $R F S$ relapse-free survival, $H R$ hazard ratio, $\mathrm{Cl}$ confidence interval

such as: (1) genes (Wnt2B and Wnt9A) of Wnt signaling pathway involved in leukemogenesis; (2) independent adverse prognostic factors in AML including WT1,
miR-155HG [3, 4], SOCS2 [15], TCF4 [16], MAP7 [17], ID1 [18] and MSI2 [19]. However, some tumor suppressors were down-regulated, such as: (1) $C D H 13$, silenced by aberrant promoter methylation, similar silencing had been found to be involved in the pathogenesis in chronic myeloid leukemia (CML) [20]; (2) VSTM1, which had also been found down-regulated in bone marrow cells from leukemia patients and played an important role in the pathogenesis of leukemia [21]; (3) CEBPA-dependent $H K 3$ expression, its decrease promoted primary AML [22]; (4) Fez1, its absence impaired Cdk1/Cdc25C interaction during mitosis and in mouse models could predispose mice to cancer development [23]; (5) TGM3, a candidate tumor suppressor gene that contributed to human head and neck cancer [24]; (6) ITPKA, its downregulation by early aberrant DNA methylation was also found in a mouse model of acute myeloid leukemia [25].

\section{Associations between genome-wide microRNA profiles and ETS2 expression}

An analysis of microRNA genome-wide profiles revealed 145 microRNAs that were strongly associated with ETS2 expression $(P<0.05$, Fig. $3 \mathrm{c})$. ETS $2^{\text {high }}$ was positively correlated with levels of miR-10a, miR-155, miR-146b and $m i R-1$. Notably, in the profiles we generated, miR-155-3p and $m i R-155-5 p$ were up-regulated (Fig. $3 \mathrm{~d}$ ). In previous reports, these microRNAs were shown to have important tumor-promoting properties. For example, overexpression of miR-10a was associated with poor OS in AML 
a

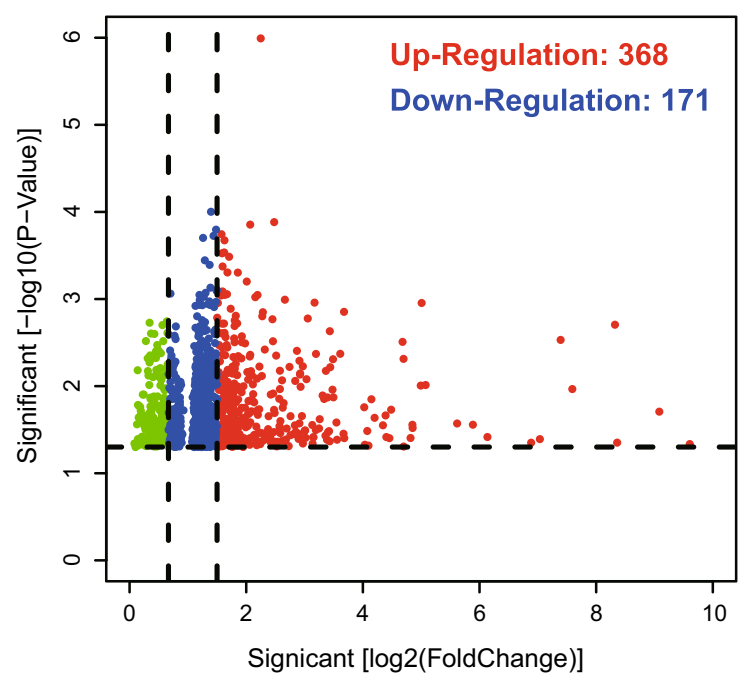

C

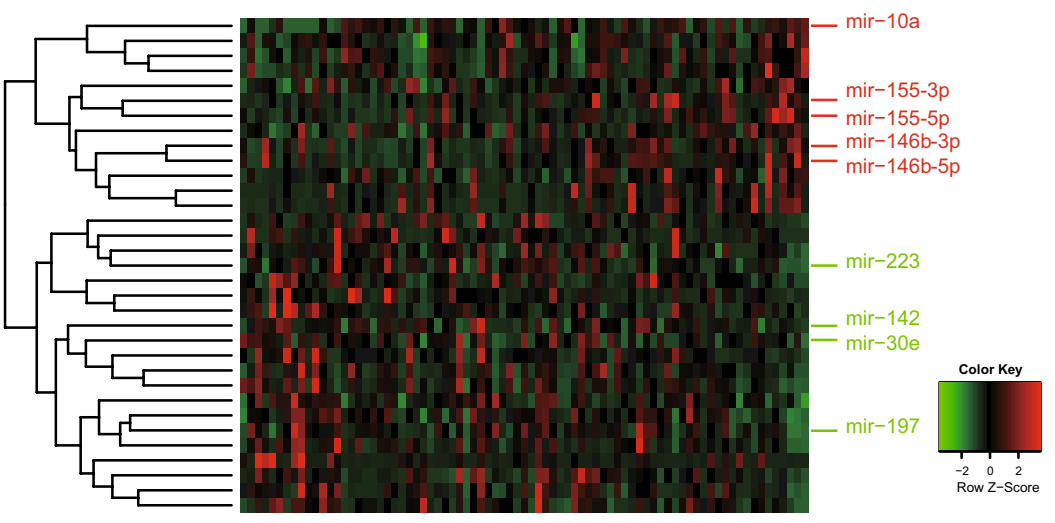

b

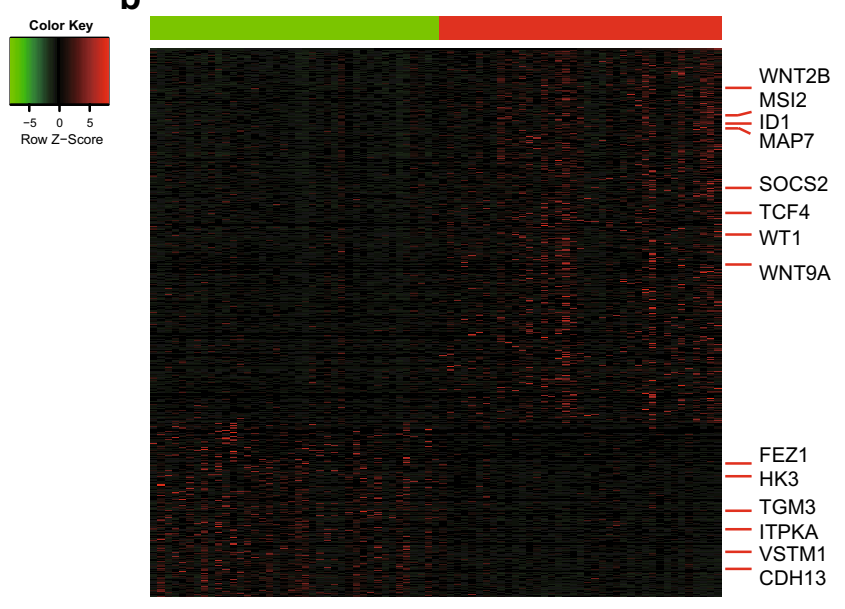

d

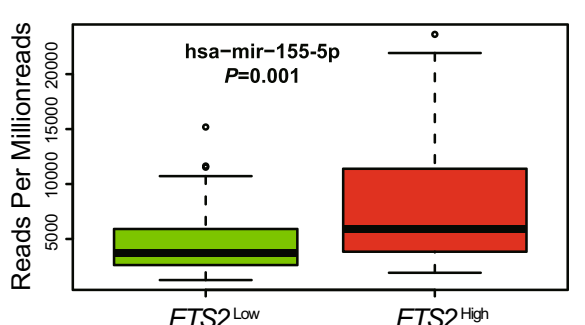

ETS2 ${ }^{\text {Low }}$

ETS2 ${ }^{\text {High }}$

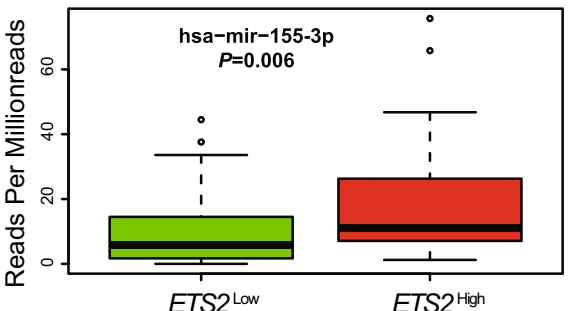

Fig. 3 Genome-wide genes/microRNAs associated with ETS2 expression. a Volcano plot of different gene-expression profiles between ETS2 ${ }^{\text {high }}$ and ETS2 ${ }^{\text {low; }}$ ETS2 ${ }^{\text {high }}$ and ETS2 ${ }^{\text {low }}$ were marked by red and green circles, respectively. $\mathbf{b}$ Expression heatmap of ETS2-associated genes. $\mathbf{c}$ Expression heatmap of associated microRNAs. Patients are ordered from left to right by increasing ETS2 expression. Expression values of the gene and microRNA probe sets are represented by color, with green indicating expression less than and red indicating expression greater than the value for the given gene and microRNA probe set. For the gene and miR-RNA expression heat map, up- and down-regulated genes and miR-RNAs mentioned in the text are indicated. $\mathbf{d}$ Boxplots of miR-155-5p and miR-155-3p expression associated with ETS2 expression

patients [26]. Up-regulation of $m i R-155$ was an independent risk factor associated with an unfavorable clinical outcome in cytogenetically normal-AML (CN-AML) [3]. Knockdown of endogenous miR-146b would result in increased transcription of tumor suppressors and inhibition of cell proliferation in chronic lymphocytic leukemia (CLL) [27]. MiR-1-2 modulation was vital for EVI1-associated tumor proliferation in acute myeloid leukemia [28]. $E T S 2^{\text {high }}$ was negatively correlated with levels of $m i R$ 223, miR-142, miR-30e and miR-197. These microRNAs had been shown to exhibit tumor suppressive properties. Low $m i R-223$ expression was associated with worse outcome in AML [29]. MiR-142-3p was a key regulator of normal myeloid differentiation; its reduced expression was involved in the leukemogenesis of AML [30]. $M i R-30 e$ induced apoptosis and could sensitize cell lines to imatinib via regulation of the BCR-ABL protein [31]. MiR-197 induced apoptosis and suppressed multiple myeloma by targeting MCL-1 [32].

\section{Association between ETS2 $2^{\text {high }}$ and adverse outcomes was confirmed by the second cohort}

We studied the second cohort of 329 previously untreated AML patients. Firstly, ETS2 $2^{\text {high }}$ AML patients $(\mathrm{n}=164)$ had significantly shorter OS $(P=0.006$, Fig. 4a) and EFS $(P=0.001$, Fig. $4 \mathrm{~b})$ than ETS2 ${ }^{\text {low }}$ patients $(\mathrm{n}=165)$. 

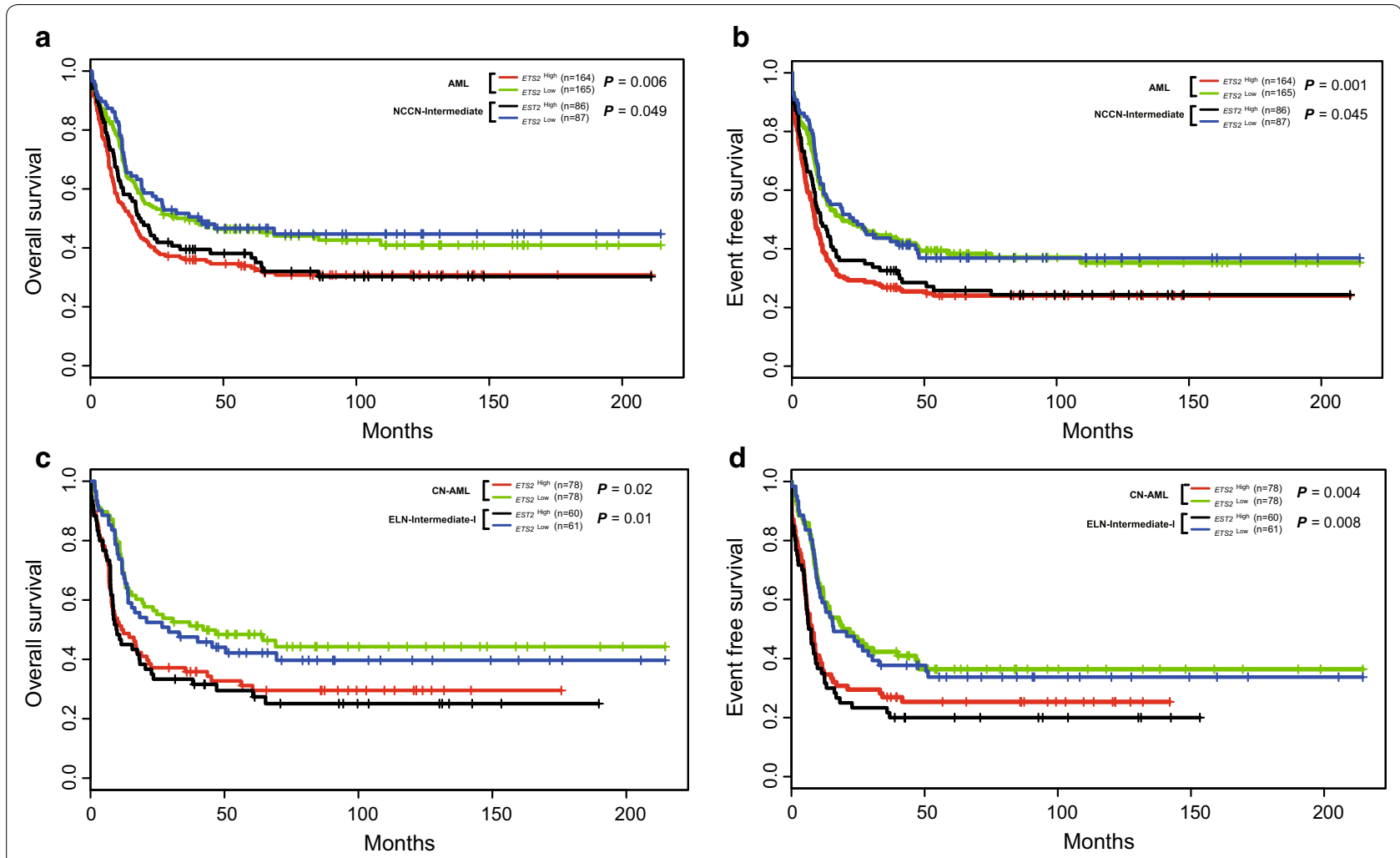

Fig. 4 The prognostic value of ETS2 expression in the second cohort. a OS and $\mathbf{b}$ EFS of 329 AML patients and the subgroup of 173 patients with NCCN intermediate-risk. c OS and d EFS of the 156 CN-AML patients and 121 AML patients in the ELN Intermediate-I category

Secondly, in the NCCN intermediate-risk AML patients, $E T S 2^{\text {high }}(\mathrm{n}=86)$ also had significantly shorter OS $(P=0.049$, Fig. 4a) and EFS $(P=0.045$, Fig. $4 \mathrm{~b})$ than ETS2 $2^{\text {low }}$ patients $(\mathrm{n}=87)$. Thirdly, ETS $2^{\text {high }} \mathrm{CN}-\mathrm{AML}$ patients $(\mathrm{n}=78)$ had significantly shorter OS $(P=0.02$, Fig. 4c) and EFS $(P=0.004$, Fig. $4 d)$ than $E T S 2^{\text {low }}$ patients $(\mathrm{n}=78)$. Fourthly, for patients in the European Leukemia Net (ELN) Intermediate-I category, ETS2 $2^{\text {high }}(\mathrm{n}=60)$ also had significantly shorter OS $(P=0.01$, Fig. $4 \mathrm{c})$ and EFS $\left(P=0.008\right.$, Fig. 4 d) than ETS $2^{\text {low }}$ patients $(\mathrm{n}=61)$.

\section{Discussion}

Identifying the prognostic factors for AML is important for the development of new targeted therapies and riskstratified treatment strategies. Recent studies had shown that high expression of ERG and ERG amplification, the most frequent copy-number alteration (CNA), are all the worse prognostic markers in AML patients [5, 6, 33]. ETS2, one of the members of the ETS family as ERG, was previously characterized as a proto-oncogene in AMKL children that is Down-syndrome and non-Downsyndrome-related [10], but the expression and clinical prognosis of ETS2 in AML remains unknown. Here, we have demonstrated the aberrant expression of ETS2 in AML patients. First, we found that ETS2 expression was up-regulated in AML cohorts and was overexpressed in the NCCN intermediate- and poor-risk groups of patients, compared to the good-risk group. These findings indicated that ETS2 might promote leukemogenesis. We also found that ETS2 showed higher expression in monocytes using publicly available expression data which suggest that ETS2 might play an important role in the function of monocytes [34] (Additional file 1: Figure S1). Second, in the first cohort, our study demonstrated that ETS2 $2^{\text {high }}$ was associated with shorter OS and EFS. Notably, ETS2 $2^{\text {high }}$ patients had longer OS and EFS after receiving allogeneic $\mathrm{HCT}$ than chemotherapy-only, but similar differences between treatment modules were not observed in ETS2 ${ }^{\text {low }}$ patients. Its presence may direct treatment decisions towards allogeneic HCT.

To further confirm the prognostic significance of ETS2, we analyzed the second cohort of uniformly treated AML patients. ETS $2^{\text {high }}$ also acted as an independent poor prognostic factor in the entire cohort, NCCN Intermediate-risk subgroup, $\mathrm{CN}$-AML subgroup, as well as the ELN Intermediate-I subgroup. The above results denoted that ETS2 $2^{\text {high }}$ was an independent, poor prognostic factor in AML. It could be employed to improve the risk stratification of ELN Intermediate-I category and NCCN Intermediate-Risk group. 
Gene and microRNA-expression profiles derived from the first cohort gave us some insight regarding the role of ETS2 in AML leukemogenesis. Tumor protein 53 (TP53) is one of the most frequently inactivated tumor suppressor genes in human cancer and its mutations predict a poor prognosis in patients with acute myeloid leukemia (AML) [35]. Recent studies have shown that mutations in the TP53 (mTP53) protects ETS2 from degradation and mTP53 disrupts ETS family target gene regulation, promoting cancer [36]. In our study, we found that ETS2 $2^{\text {high }}$ was associated with mTP53.

The expression of miR-155 has been found to be independently associated with poor clinical outcome in AML $[3,4]$. In addition, we found that $E T S 2^{\text {high }}$ was associated with over-expression of miR-155HG, miR-155-3p and $m i R-155-5 p$. This result is in accordance with recent studies which have found that ETS2 is an important transcription factor regulating $m i R-155$ [37].

\section{Conclusions}

In summary, ETS2 $2^{\text {high }}$ is an independent poor prognostic factor in AML patients and its presence should favor allogeneic HCT over chemotherapy-only in AML. In AML patients, distinctive gene/microRNA expression profiles associated with ETS2 expression may explain the role of ETS2 in the leukemogenic process.

\section{Additional file}

Additional file 1. The hierarchical differentiation tree of relationship between ETS2 expression level and hematopoietic cell differentiation.

\section{Abbreviations}

ETS: E26 transformation-specific; ETS2: V-ets avian erythroblastosis virus E26 oncogene homolog 2; AML: acute myeloid leukemia; AMKL: acute megakaryocytic leukemia; OS: overall survival; EFS: event-free survival; RFS: relapse-free survival; HCT: hematopoietic cell transplantation; FAB: French-American-British classification; FLT3-ITD: internal tandem duplication of the FLT3 gene; MLL-PTD: partial tandem duplication of the MLL gene; HR: hazard ratio; Cl: confidence interval.

\section{Authors' contributions}

$J S, L F, K X$ and XK designed the study and wrote the manuscript. HF, QW and YP performed statistical analyses. KX, LZ and JQ analyzed the data. XK, KX and JS coordinated the study over the entire time. All authors read and approved the final manuscript.

\section{Author details}

${ }^{1}$ Department of Hematology and Lymphoma Research Center, Peking University, Third Hospital, Beijing 100191, China. ${ }^{2}$ Department of Hematology, The Affiliated Hospital of Xuzhou Medical University, Xuzhou 221002, China. ${ }^{3}$ Department of Hematology, Huaihe Hospital of Henan University, Kaifeng 475000, China. ${ }^{4}$ Department of Nuclear Medicine, Chinese PLA General Hospital, Beijing 100853, China. ${ }^{5}$ Department of Medicine, Wil-liam Beaumont Hospital, Royal Oak, Ml 48073, USA. ${ }^{6}$ Northeastern University, Boston, MA 02115, USA. ${ }^{7}$ Department of Hematology, Chinese PLA General Hospital, Beijing 100853, China. ${ }^{8}$ Department of Biomedical Engineering, Chinese PLA General Hospital, Beijing 100853, China. ${ }^{9}$ Department of Medical Big Data, Chinese PLA General Hospital, Beijing 100853, China.

\section{Acknowledgements}

The authors thank all of the doctors at the institute who participated in this study for providing the follow-up samples and information.

\section{Competing interests}

The authors declare that they have no competing interests.

\section{Availability of data and materials}

The datasets of this article were generated by TCGA and GEO dataset.

\section{Ethics approval and consent to participate}

Written informed consent was obtained from all patients, and was approved by the human studies committee at Washington University.

\section{Funding}

This work was supported by grants from the National Natural Science Foundation of China $(81500118,61501519)$, the China Postdoctoral Science Foundation funded project (Project No. 2016M600443) and PLAGH project of Medical Big Data (Project No. 2016MBD-025).

\section{Publisher's Note}

Springer Nature remains neutral with regard to jurisdictional claims in published maps and institutional affiliations.

Received: 25 February 2017 Accepted: 4 July 2017

Published online: 19 July 2017

\section{References}

1. Dohner H, Weisdorf DJ, Bloomfield CD. Acute myeloid leukemia. N Engl J Med. 2015;373(12):1136-52

2. Lyu X, et al. Overexpression of Wilms tumor 1 gene as a negative prognostic indicator in acute myeloid leukemia. PLOS ONE. 2014;9(3):e92470.

3. Marcucci G, et al. Clinical role of microRNAs in cytogenetically normal acute myeloid leukemia: miR-155 upregulation independently identifies high-risk patients. J Clin Oncol. 2013;31(17):2086-93.

4. $\mathrm{Xu} \mathrm{LH}$, et al. Overexpressed miR-155 is associated with initial presentation and poor outcome in Chinese pediatric acute myeloid leukemia. Eur Rev Med Pharmacol Sci. 2015:19(24):4841-50.

5. Marcucci G, et al. Overexpression of the ETS-related gene, ERG, predicts a worse outcome in acute myeloid leukemia with normal karyotype: a cancer and leukemia group B study. J Clin Oncol. 2005;23(36):9234-42.

6. Schwind S, et al. BAALC and ERG expression levels are associated with outcome and distinct gene and microRNA expression profiles in older patients with de novo cytogenetically normal acute myeloid leukemia: a cancer and leukemia group B study. Blood. 2010;116(25):5660-9.

7. Langer $C$, et al. Prognostic importance of MN1 transcript levels, and biologic insights from MN1-associated gene and microRNA expression signatures in cytogenetically normal acute myeloid leukemia: a cancer and leukemia group B study. J Clin Oncol. 2009;27(19):3198-204.

8. Wasylyk B, Hagman J, Gutierrez-Hartmann A. Ets transcription factors: nuclear effectors of the Ras-MAP-kinase signaling pathway. Trends Biochem Sci. 1998;23(6):213-6.

9. Baldus $C D$, et al. Acute myeloid leukemia with complex karyotypes and abnormal chromosome 21: amplification discloses overexpression of APP, ETS2, and ERG genes. Proc Natl Acad Sci USA. 2004;101(11):3915-20.

10. GeY, et al. The role of the proto-oncogene ETS2 in acute megakaryocytic leukemia biology and therapy. Leukemia. 2008;22(3):521-9.

11. Santoro A, et al. Amplification of ETS2 oncogene in acute nonlymphoblastic leukemia with $\mathrm{t}(6 ; 21 ; 18)$. Cancer Genet Cytogenet. 1992;58(1):71-5.

12. Kabbout $\mathrm{M}$, et al. ETS2 mediated tumor suppressive function and MET oncogene inhibition in human non-small cell lung cancer. Clin Cancer Res. 2013;19(13):3383-95

13. The Cancer Genome Atlas Research Network. Genomic and epigenomic landscapes of adult de novo acute myeloid leukemia. N Engl J Med. 2013:368(22):2059-74. 
14. Verhaak RG, et al. Prediction of molecular subtypes in acute myeloid leukemia based on gene expression profiling. Haematologica. 2009;94(1):131-4.

15. Laszlo GS, et al. High expression of suppressor of cytokine signaling-2 predicts poor outcome in pediatric acute myeloid leukemia: a report from the Children's Oncology Group. Leuk Lymphoma. 2014;55(12):2817-21.

16. van der Reijden BA, Monteferrario D, Jansen JH, Huls G. High expression of transcription factor 4 (TCF4) is an independent adverse prognostic factor in acute myeloid leukemia that could guide treatment decisions. Haematologica. 2014;99(12):e257-9.

17. Fu L, et al. High expression of MAP7 predicts adverse prognosis in young patients with cytogenetically normal acute myeloid leukemia. Sci Rep. 2016;6:34546.

18. Zhou JD, et al. Clinical significance of up-regulated ID1 expression in Chinese de novo acute myeloid leukemia. Int J Clin Exp Pathol. 2015;8(5):5336-44.

19. Byers RJ, et al. MSI2 protein expression predicts unfavorable outcome in acute myeloid leukemia. Blood. 2011;118(10):2857-67.

20. Roman-Gomez J, et al. Cadherin-13, a mediator of calcium-dependent cell-cell adhesion, is silenced by methylation in chronic myeloid leukemia and correlates with pretreatment risk profile and cytogenetic response to interferon alfa. J Clin Oncol. 2003;21(8):1472-9.

21. Zhou J, et al. Synergistic antitumor activity of triple-regulated oncolytic adenovirus with VSTM1 and daunorubicin in leukemic cells. Apoptosis. 2016;21(10):1179-90.

22. Federzoni EA, et al. CEBPA-dependent HK3 and KLF5 expression in primary AML and during AML differentiation. Sci Rep. 2014;4:4261.

23. Vecchione $\mathrm{A}$, et al. Fez1/Lzts 1 absence impairs $\mathrm{Cdk} 1 / \mathrm{Cdc} 25 \mathrm{C}$ interaction during mitosis and predisposes mice to cancer development. Cancer Cell. 2007;11(3):275-89.

24. Wu X, et al. TGM3, a candidate tumor suppressor gene, contributes to human head and neck cancer. Mol Cancer. 2013;12(1):151.

25. Sonnet $\mathrm{M}$, et al. Early aberrant DNA methylation events in a mouse model of acute myeloid leukemia. Genome Med. 2014;6(4):34.
26. Zhi Y, et al. Serum level of miR-10-5p as a prognostic biomarker for acute myeloid leukemia. Int J Hematol. 2015;102(3):296-303.

27. Saleh LM, et al. Ibrutinib downregulates a subset of miRNA leading to upregulation of tumor suppressors and inhibition of cell proliferation in chronic lymphocytic leukemia. Leukemia. 2017;31:340-49.

28. Gomez-Benito M, et al. EVI1 controls proliferation in acute myeloid leukaemia through modulation of miR-1-2. Br J Cancer. 2010;103(8):1292-6.

29. Gentner B, et al. MicroRNA-223 dose levels fine tune proliferation and differentiation in human cord blood progenitors and acute myeloid leukemia. Exp Hematol. 2015;43(10):858-868.e7.

30. Wang XS, et al. MicroRNA-29a and microRNA-142-3p are regulators of myeloid differentiation and acute myeloid leukemia. Blood. 2012;119(21):4992-5004.

31. Hershkovitz-Rokah O, et al. MiR-30e induces apoptosis and sensitizes $\mathrm{K} 562$ cells to imatinib treatment via regulation of the BCR-ABL protein. Cancer Lett. 2015;356(2 Pt B):597-605.

32. Yang $Y$, et al. miR-137 and miR-197 induce apoptosis and suppress tumorigenicity by targeting MCL-1 in multiple myeloma. Clin Cancer Res. 2015;21(10):2399-411.

33. Nibourel $\mathrm{O}$, et al. Copy-number analysis identified new prognostic marker in acute myeloid leukemia. Leukemia. 2017;31(3):555-64.

34. Corces MR, et al. Lineage-specific and single-cell chromatin accessibility charts human hematopoiesis and leukemia evolution. Nat Genet. 2016;48(10):1193-203.

35. Kadia TM, et al. TP53 mutations in newly diagnosed acute myeloid leukemia: clinicomolecular characteristics, response to therapy, and outcomes. Cancer. 2016;122(22):3484-91.

36. Martinez LA. Mutant p53 and ETS2, a tale of reciprocity. Front Oncol. 2016;6:35.

37. Quinn SR, et al. The role of Ets2 transcription factor in the induction of microRNA-155 (miR-155) by lipopolysaccharide and its targeting by interleukin-10. J Biol Chem. 2014;289(7):4316-25.

\section{Submit your next manuscript to BioMed Central and we will help you at every step:}

- We accept pre-submission inquiries

- Our selector tool helps you to find the most relevant journal

- We provide round the clock customer support

- Convenient online submission

- Thorough peer review

- Inclusion in PubMed and all major indexing services

- Maximum visibility for your research

Submit your manuscript at www.biomedcentral.com/submit
O Biomed Central 